BIOMEDICAL AND BIOSOCIAL ANTHROPOLOGY
$\begin{gathered}\text { Official Journal of the International Academy } \\ \text { of Integrative Anthropology } \\ \text { journal homepage: http://bba-journal.com }\end{gathered}$

\title{
Correlations of linear sizes of molars with cephalometric indicators of practically healthy men of the southern region of Ukraine
}

Soboń J. S. ${ }^{1}$, Cherkasova O. V. ${ }^{2}$, Gunas V. I. ${ }^{3}$ Babych L. V. ${ }^{3}$, Kotsyura O. O.

${ }^{1}$ The Jacob of Paradies University, Gorzow Wielkopolski, Poland

${ }^{2}$ Bogomolets National Medical University, Kyiv, Ukraine

${ }^{3}$ National Pirogov Memorial Medical University, Vinnytsya, Ukraine

\author{
ARTICLE INFO \\ Received: 14 August, 2020 \\ Accepted: 16 September, 2020 \\ UDC: $611.314: 616.714 .1-071.3: 79$ \\ 055.1(477)
}

\section{CORRESPONDING AUTHOR}

e-mail: freekozak1@gmail.com Gunas V. I.

\begin{abstract}
The high prevalence of pathologies of the dental and tooth-jaw system both in Ukraine and abroad requires scientists to search for new, fundamental discoveries, in particular, to identify patterns of the relationship between cephalometric and odontometric indicators within certain ethnic populations. The purpose of the study is to establish the features of the correlations of computed tomography sizes of molars with cephalometric parameters of practically healthy men in the southern region of Ukraine. 33 practically healthy men of the first mature age, inhabitants of the southern region of Ukraine (from Mykolayiv, Kherson, Odesa, Zaporizhia regions and the Autonomous Republic of Crimea) underwent cephalometry and cone-beam computed tomography followed by odontometry of molars. The correlations between odonto- and cephalometric parameters were assessed in the license package "Statistica 6.1" using non-parametric Spearman statistics. In almost healthy men of the southern region of Ukraine in the qualitative analysis of multiple nature, mostly direct reliable and medium unreliable correlations, found only between most sizes of molars on the lower jaw and the length and height of the nose. Quantitative analysis revealed the most significant correlations between computed tomography sizes of molars and face sizes (7.4\% on the upper jaw, most of which are inverse, and $13.2 \%$ on the lower jaw, almost evenly straight and inverse). With the indicators of the skull, the relative majority of reliable, mostly inverse correlations are observed with the height of the teeth, their crowns and the length of the roots (on the upper jaw $11.4 \%$, on the lower jaw $5.4 \%$ ); and with indicators of the facial skull - almost evenly with the height of the teeth, their crowns and the length of the roots (on the upper jaw $6.4 \%$, on the lower jaw $14.6 \%$, in both cases mostly reverse) and with vestibular-lingual and mesio-distal dimensions (on the upper jaw $8.7 \%$, mostly reverse, on the lower jaw $11.8 \%$, mostly straight). The obtained results of correlation analysis of computed tomography sizes of molars with cephalometric indicators and indices confirm the population specificity of the features of the dental system and are necessary for the correct construction of regression models.
\end{abstract}

Keywords: correlations, computed tomography, odontometry, cephalometry, administrative-territorial regions of Ukraine.

\section{Introduction}

Significant scientific and technological progress that has undergone the field of dentistry, has made it one of the most developed both scientifically and technically. However, pathology of the dental and tooth-jaw system still remains a common group of diseases both in Ukraine and abroad. A survey of children attending schools in New South Wales (Australia) found that $5.14 \%$ of people had dental disease, namely: agenesis in $4.28 \%$, inclination in $0.6 \%$ and the presence of excess teeth in $0.28 \%$ [8]. A survey conducted in
India found the following data: $36.7 \%$ of patients had at least one dental abnormality, among which the most common were adentia $16.3 \%$, inclination $15.5 \%$, the presence of excess teeth $1.2 \%$ and microdontia in $1.0 \%$ of subjects [15]. Data from Thailand show slightly different figures - the most common anomalies in this region are congenital adentia (found in $34.38 \%$ ), followed by the presence of excess teeth $(20.31 \%)$ and a slightly lower percentage of people with inclination of teeth $(9.38 \%)$ [22]. The analysis 
conducted in only one French hospital for the period 20032013 revealed that almost half of the patients seeking medical help had at least one dental-maxillary pathology [3].

Given that the subjects in different parts of the world have a fairly high frequency of detection of pathologies of the dental and tooth-jaw system, it would be appropriate to focus research efforts on creating algorithms that could be used for the purpose of screening examination of persons to identify predisposition to these pathologies, taking into account various variables, namely: age, sex, ethnicity and regional affiliation, which would have the ultimate goal to ensure the full aesthetic function and operation of the dental apparatus among persons at risk.

Given the results of studies by foreign authors from around the world [9, 14, 20, 24], which identified and proved the existence of links between cephalometric indicators and odontometric indicators for the local population, and more, the detection of sexual dimorphism, it is appropriate application of anthropology in order to identify the relationship between the size of the cerebral and facial skull and certain indicators of the dental and maxillofacial apparatus for the population of Ukraine, taking into account their regional affiliation and gender.

That is why the purpose of our study is to establish the features of the correlations of computed tomography size of molars with cephalometric parameters of practically healthy men in the southern region of Ukraine.

\section{Materials and methods}

The study selected 33 somatically healthy men, residents of the southern region of Ukraine (from Mykolayiv, Kherson, Odessa, Zaporizhia regions and the Autonomous Republic of Crimea) with favorable, moderately favorable and satisfactory ecological living conditions according to research by the National Ecological Center of Ukraine (http:/ /superdom.ua/view/1454-ekologicheskaya-karta-ukrainygde-luchshe-zhit.html). All selected men underwent computed tomography using a Veraviewepocs-3D dental cone-beam tomograph (Morita, Japan) and cephalometry. Committee on Bioethics of National Pirogov Memorial
Medical University, Vinnytsya found that the study does not contradict the basic bioethical norms of the Council of Europe Convention on Human Rights and Biomedicine (1977), the Declaration of Helsinki, and meet ethical and moral requirements under the order of the Ministry of Health of Ukraine № 281 from 01.11.2000.

Odontometric examination of the dental-jaw complex in three projections was performed in the software shell i-Dixel One Volume Viewer (Ver.1.5.0. J Morita Mfg. Cor.). Measurements of the first and second molars (M) of the right and left sides of the upper and lower jaws were performed: height of the tooth and crown of the tooth, length of the palatal medial and distal roots, vestibular-lingual and mesio-distal dimensions of the crown and neck of the tooth [23]. The study used digital notation of teeth: 16 - upper right first $M ; 17$ - upper right second $M ; 26$ - upper left first $M ; 27$ upper left second $M ; 36$ - lower left first $M ; 37$ - lower left second $\mathrm{M} ; 46$ - lower right first $\mathrm{M} ; 47$ - lower right second $\mathrm{M}$.

Cephalometric study was performed in accordance with generally accepted recommendations and anatomical guidelines and points [6]. It involved measuring the parameters of the cerebral and facial parts of the head with a large Martin compass and a soft centimeter tape.

The shape of the head was determined by the ratio of the largest width of the head to the length of the head, and according to the obtained value of the indicator was the distribution of craniotypes [26]. The type of face was calculated using the Garson index (the ratio of morphological length of the face to the largest width of the face), and according to the obtained value of the indicator was the distribution of face types [19].

In the license package "Statistica 6.1" the correlations between odonto- and cephalometric parameters were estimated using non-parametric Spearman's statistics.

\section{Results}

Correlations between computed tomographic linear dimensions of the upper and lower jaws $M$ with cephalometric parameters of the brain and facial skull of practically healthy men of the first mature age, residents of

Table 1. Correlations of the sizes of $M$ of an upper jaw with cephalometric indicators of a brain skull of men of the southern region of Ukraine $(n=6-33)$.

\begin{tabular}{|c|c|c|c|c|c|c|c|}
\hline \multirow{2}{*}{ Sizes of M } & \multicolumn{9}{|c|}{ Cephalometric indicators } \\
\cline { 2 - 8 } & DUG_GOP & DUGS_GOP & DUG_AUAU & G_OP & FMT_FMT & EY_EY & KRANIO \\
\hline 17HZ & -0.03 & 0.06 & -0.14 & 0.18 & 0.01 & -0.30 & $\underline{-0.31}$ \\
\hline 17HKZ & 0.03 & 0.11 & 0.13 & 0.09 & -0.32 & -0.25 & -0.23 \\
\hline 17HRZ1 & 0.09 & 0.17 & 0.10 & 0.24 & -0.25 & -0.24 & -0.38 \\
\hline 17HRZ2 & 0.10 & 0.28 & -0.01 & 0.17 & 0.03 & -0.28 & -0.28 \\
\hline 17HRZ3 & -0.21 & -0.20 & -0.15 & -0.25 & 0.16 & -0.06 & 0.14 \\
\hline 17VO_K & -0.15 & -0.11 & 0.12 & -0.03 & -0.23 & -0.13 & -0.04 \\
\hline 17VO_S & -0.05 & -0.15 & 0.09 & -0.09 & -0.24 & -0.06 & -0.03 \\
\hline 17MD_K & -0.12 & -0.17 & -0.01 & -0.02 & 0.28 & -0.20 & -0.11 \\
\hline
\end{tabular}


Continuation of table 1.

\begin{tabular}{|c|c|c|c|c|c|c|c|}
\hline \multirow{2}{*}{ Sizes of $M$} & \multicolumn{7}{|c|}{ Cephalometric indicators } \\
\hline & DUG_GOP & DUGS_GOP & DUG_AUAU & G_OP & FMT_FMT & EY_EY & KRANIO \\
\hline 17MD_S & -0.12 & -0.16 & -0.03 & 0.09 & 0.23 & -0.26 & -0.22 \\
\hline $16 \mathrm{HZ}$ & -0.28 & -0.07 & -0.02 & -0.33 & 0.13 & -0.03 & 0.21 \\
\hline $16 \mathrm{HKZ}$ & -0.14 & 0.18 & -0.03 & -0.17 & 0.06 & -0.11 & 0.11 \\
\hline 16HRZ1 & -0.23 & -0.20 & 0.00 & -0.34 & -0.05 & -0.05 & 0.20 \\
\hline 16HRZ2 & -0.01 & -0.05 & -0.10 & 0.01 & 0.34 & 0.05 & 0.01 \\
\hline 16HRZ3 & -0.11 & 0.12 & 0.03 & -0.20 & -0.07 & 0.03 & 0.16 \\
\hline 16VO_S & -0.04 & 0.00 & -0.02 & 0.22 & -0.11 & -0.01 & -0.19 \\
\hline 16VO_K & -0.15 & -0.07 & -0.12 & 0.09 & 0.08 & -0.11 & -0.12 \\
\hline 16MD_S & -0.21 & -0.19 & 0.07 & -0.01 & -0.06 & -0.17 & -0.12 \\
\hline 16MD K & -0.18 & -0.20 & 0.11 & 0.04 & 0.11 & -0.24 & -0.19 \\
\hline $26 \mathrm{HZ}$ & -0.20 & -0.02 & 0.03 & -0.15 & 0.12 & -0.05 & 0.05 \\
\hline $26 \mathrm{HKZ}$ & -0.07 & 0.20 & 0.01 & -0.07 & 0.09 & -0.17 & -0.04 \\
\hline $26 \mathrm{HRZ1}$ & -0.41 & -0.18 & -0.07 & -0.07 & -0.12 & -0.42 & -0.25 \\
\hline 26HRZ2 & -0.17 & -0.10 & -0.05 & -0.16 & 0.20 & 0.01 & 0.12 \\
\hline $26 \mathrm{HRZ3}$ & -0.03 & 0.15 & 0.01 & -0.15 & -0.06 & 0.06 & 0.12 \\
\hline 26VO_K & -0.15 & -0.08 & -0.12 & 0.08 & -0.01 & -0.02 & -0.10 \\
\hline 26VO_S & -0.13 & -0.07 & -0.06 & 0.17 & 0.03 & -0.14 & -0.20 \\
\hline 26MD_K & -0.35 & -0.22 & 0.04 & 0.01 & -0.19 & -0.37 & -0.25 \\
\hline 26MD_S & -0.30 & -0.31 & 0.03 & 0.03 & -0.08 & -0.27 & -0.20 \\
\hline $27 \mathrm{HZ}$ & -0.09 & 0.05 & -0.17 & 0.13 & -0.03 & -0.31 & -0.27 \\
\hline $27 \mathrm{HKZ}$ & -0.07 & 0.09 & 0.10 & 0.02 & -0.41 & -0.27 & -0.19 \\
\hline 27HRZ1 & 0.07 & 0.10 & 0.11 & 0.18 & -0.35 & -0.32 & -0.38 \\
\hline 27HRZ2 & -0.03 & 0.14 & 0.00 & 0.19 & 0.12 & -0.34 & -0.34 \\
\hline 27HRZ3 & -0.29 & -0.27 & -0.16 & -0.28 & 0.16 & -0.10 & 0.13 \\
\hline 27VO_K & -0.13 & -0.13 & 0.06 & 0.06 & -0.17 & -0.23 & -0.21 \\
\hline 27VO_S & -0.10 & -0.20 & 0.13 & 0.03 & -0.30 & -0.18 & -0.19 \\
\hline 27MD_K & -0.13 & -0.20 & -0.09 & 0.00 & 0.16 & -0.15 & -0.11 \\
\hline $27 \mathrm{MD} \mathrm{S}$ & -0.17 & -0.22 & -0.13 & 0.01 & 0.11 & -0.22 & -0.16 \\
\hline
\end{tabular}

Notes: here and in the following tables, bold and red highlighted reliable medium-strength direct correlations; bold and blue highlighted significant medium-strength inverse correlations; underlining and green highlight inaccurate medium-strength inverse correlations; DUG_GOP - the largest head circumference; DUGS_GOP - sagittal arch; DUG_AUAU - transverse arc; G_OP - the largest length of the head; FMT_FMT - the smallest width of the head (frontal diameter); EU_EU - the largest width of the head (occipital diameter); KRANIO - cranial index; HZ - tooth height; HKZ - height of the tooth crown; HRZ1 - length of the palatine root of the upper M; HRZ2 - length of the dorsal proximal root of the upper M; HRZ3 - length of the vestibular distal root of the upper M; VO_K - vestibular-lingual size of the tooth crown; VO_S - vestibular-lingual size of the neck of the tooth; MD_K - mesio-distal size of the tooth crown; MD_S - mesio-distal size of the tooth neck.

Table 2. Correlations of the sizes of $M$ of a lower jaw with cephalometric indicators of a brain skull of men of the southern region of Ukraine $(n=6-33)$.

\begin{tabular}{|c|c|c|c|c|c|c|c|}
\hline \multirow{2}{*}{ Sizes of M } & \multicolumn{9}{|c|}{ Cephalometric indicators } \\
\cline { 2 - 9 } & DUG_GOP & DUGS_GOP & DUG_AUAU & G_OP & FMT_FMT & EY_EY & KRANIO \\
\hline $47 \mathrm{HZ}$ & -0.04 & 0.15 & 0.15 & -0.01 & -0.19 & -0.21 & -0.11 \\
\hline $47 \mathrm{HKZ}$ & -0.06 & 0.05 & 0.15 & 0.04 & -0.28 & -0.23 & -0.21 \\
\hline $47 \mathrm{HRZ4}$ & -0.07 & 0.06 & 0.17 & 0.14 & -0.33 & -0.14 & -0.30 \\
\hline 47HRZ5 & 0.00 & 0.21 & 0.13 & -0.08 & -0.04 & $\underline{-0.33}$ & 0.07 \\
\hline
\end{tabular}


Soboń J. S., Cherkasova O. V., Gunas V. I., Babych L. V., Kotsyura O. O.

Continuation of table 2.

\begin{tabular}{|c|c|c|c|c|c|c|c|}
\hline \multirow{2}{*}{ Sizes of $\mathrm{M}$} & \multicolumn{7}{|c|}{ Cephalometric indicators } \\
\hline & DUG_GOP & DUGS_GOP & DUG_AUAU & G_OP & FMT_FMT & EY_EY & KRANIO \\
\hline 47VO_K & -0.09 & -0.07 & 0.07 & -0.08 & 0.01 & 0.12 & 0.04 \\
\hline 47VO_S & -0.18 & -0.15 & -0.02 & -0.19 & -0.01 & -0.13 & 0.07 \\
\hline 47MD_K & 0.03 & 0.02 & -0.03 & 0.02 & -0.11 & -0.15 & -0.10 \\
\hline 47MD_S & 0.01 & -0.04 & -0.04 & 0.07 & -0.17 & -0.25 & -0.20 \\
\hline $46 \mathrm{HZ}$ & -0.27 & 0.04 & -0.13 & -0.14 & -0.11 & 0.19 & 0.06 \\
\hline $46 \mathrm{HKZ}$ & 0.11 & 0.26 & 0.05 & -0.04 & 0.01 & 0.08 & -0.01 \\
\hline $46 \mathrm{HRZ4}$ & -0.30 & -0.12 & -0.09 & -0.10 & -0.19 & 0.11 & -0.06 \\
\hline 46HRZ5 & -0.38 & -0.28 & -0.13 & -0.17 & -0.24 & 0.02 & 0.04 \\
\hline 46VO_K & -0.07 & 0.01 & -0.19 & -0.06 & -0.12 & 0.00 & -0.08 \\
\hline 46VO_S & 0.04 & -0.06 & 0.05 & -0.02 & -0.08 & -0.11 & -0.09 \\
\hline 46MD_K & -0.14 & 0.02 & -0.18 & -0.24 & -0.20 & -0.09 & 0.03 \\
\hline 46MD_S & 0.04 & 0.04 & 0.08 & 0.03 & -0.01 & -0.15 & -0.09 \\
\hline $36 \mathrm{HZ}$ & -0.18 & 0.12 & -0.13 & 0.02 & -0.06 & 0.11 & -0.01 \\
\hline $36 \mathrm{HKZ}$ & -0.06 & 0.17 & -0.06 & -0.03 & -0.06 & 0.00 & -0.05 \\
\hline $36 \mathrm{HRZ4}$ & -0.20 & -0.06 & -0.03 & 0.05 & -0.09 & 0.07 & -0.09 \\
\hline $36 \mathrm{HRZ5}$ & -0.28 & -0.28 & 0.00 & -0.15 & -0.12 & 0.06 & 0.09 \\
\hline 36VO_K & -0.03 & -0.09 & -0.12 & -0.01 & -0.01 & 0.07 & -0.08 \\
\hline 36VO_S & -0.15 & -0.15 & -0.14 & -0.19 & -0.11 & 0.00 & 0.04 \\
\hline 36MD_K & -0.17 & -0.03 & -0.22 & -0.24 & -0.14 & 0.06 & 0.07 \\
\hline 36MD_S & 0.00 & 0.04 & 0.03 & 0.05 & 0.00 & -0.10 & -0.09 \\
\hline $37 \mathrm{HZ}$ & -0.08 & 0.09 & 0.15 & 0.11 & -0.25 & -0.26 & -0.24 \\
\hline $37 \mathrm{HKZ}$ & -0.10 & -0.04 & 0.12 & 0.09 & -0.29 & -0.11 & -0.27 \\
\hline 37HRZ4 & -0.10 & 0.06 & 0.07 & 0.11 & -0.27 & -0.09 & -0.25 \\
\hline 37HRZ5 & 0.00 & 0.20 & 0.08 & -0.06 & -0.08 & -0.35 & 0.02 \\
\hline 37VO_K & -0.07 & -0.05 & 0.09 & -0.17 & 0.07 & 0.04 & 0.14 \\
\hline 37VO_S & -0.13 & -0.15 & -0.05 & -0.11 & -0.03 & -0.07 & 0.01 \\
\hline 37MD_K & -0.06 & -0.11 & -0.01 & -0.09 & 0.04 & -0.11 & 0.04 \\
\hline 37MD_S & 0.06 & -0.04 & -0.02 & 0.00 & -0.01 & -0.18 & -0.07 \\
\hline
\end{tabular}

Notes: here and in the following tables, HRZ4 - the length of the near root of the lower M; HRZ5 - the length of the distal root of the lower M.

Table 3. Correlations of the sizes of $M$ of an upper jaw with cephalometric indicators of a facial skull of men of the southern region of Ukraine $(n=6-33)$.

\begin{tabular}{|c|c|c|c|c|c|c|c|c|c|}
\hline \multirow{2}{*}{ Sizes of M } & \multicolumn{9}{|c|}{ Cephalometric indicators } \\
\cline { 2 - 11 } & ZY_ZY & ZM_ZM & TR_GN & TR_N & N_GN & N_PRN & N_SN & GO_GO & N_STO \\
\hline 17HZ & -0.15 & -0.09 & -0.14 & 0.03 & 0.02 & 0.22 & 0.21 & -0.15 & 0.08 \\
\hline 17HKZ & -0.32 & -0.07 & -0.06 & 0.19 & 0.01 & 0.15 & 0.13 & 0.06 & 0.08 \\
\hline 17HRZ1 & -0.30 & -0.01 & -0.19 & 0.00 & 0.08 & 0.16 & 0.18 & 0.04 & 0.08 \\
\hline 17HRZ2 & -0.15 & -0.14 & -0.11 & 0.21 & -0.02 & 0.28 & 0.29 & 0.07 & 0.16 \\
\hline 17HRZ3 & 0.16 & -0.15 & 0.08 & 0.09 & -0.10 & -0.01 & 0.01 & -0.23 & -0.06 \\
\hline 17VO_K & -0.04 & -0.06 & -0.11 & -0.19 & -0.20 & -0.08 & -0.10 & $\underline{-0.34}$ & -0.18 \\
\hline 17VO_S & -0.11 & -0.05 & -0.14 & -0.16 & -0.05 & 0.08 & 0.06 & -0.13 & -0.03 \\
\hline 17MD_K & 0.24 & -0.09 & 0.13 & 0.20 & -0.03 & $\underline{0.30}$ & $\underline{0.30}$ & -0.14 & 0.00 \\
\hline
\end{tabular}


Correlations of linear sizes of molars with cephalometric indicators of practically healthy men of the southern...

\section{Continuation of table 3.}

\begin{tabular}{|c|c|c|c|c|c|c|c|c|c|}
\hline \multirow{2}{*}{ Sizes of $M$} & \multicolumn{9}{|c|}{ Cephalometric indicators } \\
\hline & ZY_ZY & ZM_ZM & TR_GN & TR_N & N_GN & N_PRN & N_SN & GO_GO & N_STO \\
\hline 17MD_S & 0.22 & -0.09 & 0.13 & 0.16 & 0.06 & 0.35 & $\underline{0.34}$ & -0.14 & 0.13 \\
\hline $16 \mathrm{HZ}$ & 0.21 & -0.38 & $\underline{-0.31}$ & -0.01 & -0.40 & -0.02 & 0.02 & -0.21 & -0.02 \\
\hline $16 \mathrm{HKZ}$ & 0.10 & -0.21 & -0.06 & 0.29 & 0.05 & 0.18 & 0.19 & -0.17 & 0.13 \\
\hline $16 \mathrm{HRZ1}$ & -0.01 & -0.19 & -0.09 & 0.01 & -0.08 & -0.09 & -0.04 & 0.06 & 0.02 \\
\hline $16 \mathrm{HRZ2}$ & 0.25 & 0.04 & -0.22 & -0.06 & -0.37 & -0.26 & -0.28 & -0.20 & -0.19 \\
\hline $16 \mathrm{HRZ3}$ & 0.06 & -0.36 & -0.24 & 0.04 & -0.33 & 0.03 & 0.09 & -0.17 & 0.04 \\
\hline 16VO_S & 0.07 & -0.15 & -0.18 & -0.16 & -0.15 & 0.01 & 0.00 & -0.38 & -0.15 \\
\hline 16VO_K & 0.23 & -0.18 & 0.03 & 0.03 & 0.03 & 0.26 & 0.26 & -0.22 & 0.02 \\
\hline 16MD_S & -0.06 & -0.12 & -0.22 & -0.23 & -0.06 & 0.02 & 0.05 & $\underline{-0.31}$ & -0.16 \\
\hline 16MD_K & 0.10 & 0.01 & -0.14 & -0.20 & -0.08 & 0.01 & 0.02 & -0.25 & -0.22 \\
\hline $26 \mathrm{HZ}$ & 0.11 & -0.38 & -0.32 & 0.01 & -0.31 & 0.12 & 0.15 & -0.17 & 0.05 \\
\hline $26 \mathrm{HKZ}$ & 0.03 & -0.26 & -0.05 & 0.31 & 0.07 & 0.28 & 0.29 & 0.03 & 0.23 \\
\hline $26 \mathrm{HRZ1}$ & 0.06 & -0.45 & -0.27 & -0.06 & -0.01 & 0.14 & 0.16 & -0.11 & 0.15 \\
\hline 26HRZ2 & 0.09 & -0.04 & 0.00 & 0.22 & -0.21 & -0.14 & -0.16 & -0.08 & -0.17 \\
\hline $26 \mathrm{HRZ3}$ & 0.03 & -0.41 & -0.27 & -0.05 & -0.16 & 0.18 & 0.24 & -0.19 & 0.13 \\
\hline 26VO_K & 0.14 & -0.24 & -0.09 & -0.08 & -0.10 & 0.13 & 0.13 & -0.30 & -0.06 \\
\hline 26VO_S & 0.23 & -0.19 & 0.01 & 0.02 & 0.01 & 0.25 & 0.25 & -0.23 & 0.06 \\
\hline 26MD_K & -0.13 & -0.21 & -0.32 & $\underline{-0.31}$ & -0.02 & 0.09 & 0.08 & -0.42 & -0.17 \\
\hline 26MD_S & -0.02 & -0.09 & -0.26 & -0.37 & 0.05 & 0.07 & 0.05 & -0.34 & -0.15 \\
\hline $27 \mathrm{HZ}$ & -0.07 & -0.22 & -0.17 & 0.04 & -0.04 & 0.17 & 0.19 & -0.24 & 0.12 \\
\hline $27 \mathrm{HKZ}$ & -0.40 & -0.16 & -0.07 & 0.21 & 0.05 & 0.13 & 0.12 & 0.01 & 0.12 \\
\hline 27HRZ1 & -0.34 & -0.11 & -0.08 & 0.15 & 0.02 & 0.19 & 0.19 & 0.09 & 0.12 \\
\hline 27HRZ2 & 0.02 & -0.22 & -0.12 & 0.22 & -0.08 & 0.28 & $\underline{0.31}$ & -0.19 & 0.25 \\
\hline 27HRZ3 & 0.18 & -0.23 & 0.01 & 0.05 & -0.10 & -0.05 & -0.03 & -0.28 & -0.02 \\
\hline 27VO_K & 0.00 & -0.12 & -0.11 & -0.25 & -0.05 & 0.00 & -0.02 & $\underline{-0.31}$ & -0.09 \\
\hline 27VO_S & -0.15 & -0.03 & -0.04 & -0.02 & -0.02 & 0.08 & 0.05 & -0.08 & -0.01 \\
\hline 27MD_K & 0.16 & -0.16 & -0.09 & -0.04 & -0.03 & 0.29 & 0.28 & -0.18 & 0.06 \\
\hline 27MD_S & 0.12 & -0.16 & -0.07 & -0.06 & 0.06 & $\underline{0.31}$ & $\underline{0.30}$ & -0.20 & 0.11 \\
\hline
\end{tabular}

Notes: here and in the following tables, by underlining and sand color are selected unreliable medium-strength direct correlations; ZY_ZY - face width (distance between chin points); ZM_ZM - average width of the face; TR_GN - physiological length of the face; TR_N - lobe height; N_GN - morphological length of the face; N_PRN - length of the nose; N_SN - height of the nose; GO_GO - width of the lower jaw (width between the corners of the lower jaw); N_STO - height of the upper part of the face.

Table 4. Correlations of the sizes of $M$ of a mandible with cephalometric indicators of a facial skull of men of the southern region of Ukraine $(n=6-33)$

\begin{tabular}{|c|c|c|c|c|c|c|c|c|c|}
\hline \multirow{2}{*}{ Sizes of M } & \multicolumn{10}{|c|}{ Cephalometric indicators } \\
\cline { 2 - 12 } & ZY_ZY & ZM_ZM & TR_GN & TR_N & N_GN & N_PRN & N_SN & GO_GO & N_STO \\
\hline 47HZ & -0.29 & -0.24 & -0.35 & -0.08 & -0.04 & $\underline{0.30}$ & $\underline{0.34}$ & -0.18 & 0.15 \\
\hline 47HKZ & -0.35 & -0.18 & -0.24 & 0.00 & 0.10 & 0.38 & 0.40 & -0.08 & 0.22 \\
\hline 47HRZ4 & $\underline{-0.30}$ & -0.20 & -0.35 & -0.05 & -0.06 & 0.28 & 0.28 & -0.18 & 0.16 \\
\hline 47HRZ5 & -0.22 & -0.18 & $\underline{-0.33}$ & -0.15 & -0.20 & -0.09 & -0.04 & $\underline{-0.31}$ & -0.02 \\
\hline 47VO_K & 0.15 & -0.24 & -0.21 & -0.18 & -0.09 & 0.28 & $\underline{0.30}$ & -0.21 & -0.04 \\
\hline 47VO_S & 0.04 & -0.25 & -0.14 & -0.10 & 0.10 & $\underline{0.32}$ & $\underline{0.34}$ & 0.02 & 0.02 \\
\hline
\end{tabular}


Soboń J. S., Cherkasova O. V., Gunas V. I., Babych L. V., Kotsyura O. O.

Continuation of table 4.

\begin{tabular}{|c|c|c|c|c|c|c|c|c|c|}
\hline \multirow{2}{*}{ Sizes of M } & \multicolumn{9}{|c|}{ Cephalometric indicators } \\
\hline & ZY_ZY & ZM_ZM & TR_GN & TR_N & N_GN & N_PRN & N_SN & GO_GO & N_STO \\
\hline 47MD_K & -0.09 & -0.26 & -0.09 & -0.03 & 0.14 & 0.56 & 0.56 & -0.10 & 0.27 \\
\hline 47MD_S & -0.06 & -0.35 & -0.11 & -0.02 & 0.09 & 0.51 & 0.51 & -0.01 & 0.29 \\
\hline $46 \mathrm{HZ}$ & 0.06 & -0.26 & -0.02 & 0.20 & 0.06 & 0.16 & 0.18 & -0.11 & 0.10 \\
\hline $46 \mathrm{HKZ}$ & 0.01 & -0.09 & 0.03 & $\underline{0.32}$ & 0.24 & 0.29 & $\underline{0.31}$ & 0.23 & 0.14 \\
\hline 46HRZ4 & 0.08 & -0.26 & 0.12 & 0.22 & 0.28 & $\underline{0.31}$ & $\underline{0.32}$ & 0.01 & 0.20 \\
\hline 46HRZ5 & -0.07 & -0.14 & -0.23 & -0.37 & -0.25 & -0.36 & -0.36 & -0.49 & -0.11 \\
\hline 46VO_K & 0.21 & -0.36 & -0.14 & -0.20 & 0.13 & 0.43 & 0.44 & -0.19 & 0.13 \\
\hline 46VO_S & 0.05 & -0.14 & -0.02 & -0.03 & 0.08 & 0.31 & 0.32 & 0.15 & 0.19 \\
\hline 46MD_K & 0.16 & -0.39 & -0.02 & 0.00 & 0.19 & 0.53 & 0.52 & -0.14 & 0.08 \\
\hline 46MD_S & 0.01 & -0.16 & -0.12 & 0.02 & 0.10 & $\underline{0.33}$ & $\underline{0.34}$ & 0.11 & -0.02 \\
\hline $36 \mathrm{HZ}$ & 0.02 & -0.18 & -0.02 & 0.17 & 0.15 & 0.16 & 0.17 & -0.15 & 0.14 \\
\hline $36 \mathrm{HKZ}$ & 0.00 & -0.24 & -0.03 & 0.28 & 0.21 & 0.30 & 0.32 & 0.04 & 0.19 \\
\hline $36 \mathrm{HRZ4}$ & 0.00 & -0.12 & 0.08 & 0.15 & 0.25 & 0.21 & 0.21 & -0.04 & 0.18 \\
\hline $36 \mathrm{HRZ5}$ & -0.11 & 0.05 & -0.11 & -0.36 & -0.20 & -0.42 & -0.44 & -0.36 & -0.19 \\
\hline 36VO_K & 0.21 & -0.19 & -0.04 & -0.08 & -0.02 & 0.26 & 0.26 & -0.07 & -0.06 \\
\hline 36VO_S & 0.20 & $\underline{-0.32}$ & -0.15 & -0.19 & 0.16 & 0.35 & 0.36 & -0.17 & 0.17 \\
\hline 36MD_K & 0.28 & -0.45 & -0.16 & -0.08 & 0.12 & 0.49 & 0.48 & -0.22 & 0.13 \\
\hline 36MD_S & 0.05 & -0.26 & -0.25 & -0.06 & -0.01 & $\underline{0.34}$ & 0.35 & 0.02 & -0.01 \\
\hline $37 \mathrm{HZ}$ & -0.28 & -0.26 & -0.42 & -0.19 & -0.02 & 0.27 & $\underline{0.30}$ & -0.19 & 0.20 \\
\hline $37 \mathrm{HKZ}$ & -0.24 & -0.20 & -0.36 & -0.13 & 0.02 & 0.37 & 0.38 & -0.14 & 0.28 \\
\hline 37HRZ4 & -0.20 & -0.19 & -0.31 & -0.05 & 0.03 & 0.26 & 0.25 & -0.26 & 0.22 \\
\hline 37HRZ5 & -0.27 & -0.19 & -0.29 & -0.14 & -0.12 & -0.11 & -0.06 & -0.21 & 0.01 \\
\hline 37VO_K & 0.06 & -0.17 & -0.12 & -0.13 & 0.01 & 0.26 & 0.28 & -0.10 & -0.09 \\
\hline 37VO_S & 0.05 & -0.16 & -0.08 & -0.11 & 0.11 & $\underline{0.34}$ & $\underline{0.34}$ & 0.07 & -0.03 \\
\hline 37MD_K & -0.08 & -0.23 & -0.08 & 0.02 & 0.10 & 0.52 & 0.52 & -0.04 & 0.07 \\
\hline 37MD_S & -0.04 & -0.22 & -0.11 & -0.04 & 0.17 & 0.55 & 0.54 & 0.07 & 0.14 \\
\hline
\end{tabular}

Table 5. Correlations of the sizes of $\mathrm{M}$ of an upper jaw with cephalometric indicators of a facial skull of men of the southern region of Ukraine $(n=6-33)$.

\begin{tabular}{|c|c|c|c|c|c|c|c|c|c|}
\hline \multirow{2}{*}{ Sizes of $M$} & \multicolumn{9}{|c|}{ Cephalometric indicators } \\
\hline & SN_PRN & AL_AL & $\mathrm{CHI} \_\mathrm{CHI}$ & EK_EK & MF_MF & N_l & PGO_GN & IGO_GN & IN_GARS \\
\hline $17 \mathrm{HZ}$ & 0.13 & 0.02 & -0.11 & -0.21 & -0.08 & -0.06 & 0.04 & -0.11 & 0.15 \\
\hline $17 \mathrm{HKZ}$ & 0.17 & 0.07 & 0.07 & -0.14 & 0.11 & 0.17 & -0.06 & -0.20 & 0.29 \\
\hline 17HRZ1 & $\underline{0.30}$ & 0.07 & -0.05 & -0.18 & 0.03 & 0.05 & -0.10 & -0.24 & 0.26 \\
\hline 17HRZ2 & 0.14 & -0.03 & 0.16 & 0.12 & 0.07 & 0.21 & $\underline{0.32}$ & 0.19 & 0.11 \\
\hline 17HRZ3 & -0.21 & 0.25 & -0.24 & -0.15 & -0.09 & -0.12 & -0.04 & -0.15 & -0.12 \\
\hline 17VO_K & -0.20 & 0.14 & 0.02 & $\underline{-0.32}$ & -0.14 & -0.21 & -0.22 & -0.20 & -0.13 \\
\hline 17VO_S & -0.10 & 0.08 & 0.13 & $\underline{-0.32}$ & 0.10 & -0.10 & -0.11 & -0.18 & -0.06 \\
\hline 17MD_K & -0.08 & 0.16 & 0.24 & -0.04 & 0.02 & 0.02 & 0.17 & 0.08 & -0.23 \\
\hline 17MD_S & 0.03 & 0.08 & 0.26 & 0.02 & 0.11 & 0.09 & 0.18 & 0.12 & -0.21 \\
\hline $16 \mathrm{HZ}$ & -0.25 & 0.18 & -0.02 & -0.14 & -0.28 & -0.23 & -0.04 & -0.13 & -0.13 \\
\hline $16 \mathrm{HKZ}$ & 0.00 & -0.15 & 0.04 & 0.09 & -0.08 & 0.05 & 0.01 & -0.08 & 0.03 \\
\hline
\end{tabular}


Correlations of linear sizes of molars with cephalometric indicators of practically healthy men of the southern...

\section{Continuation of table 5 .}

\begin{tabular}{|c|c|c|c|c|c|c|c|c|c|}
\hline \multirow{2}{*}{ Sizes of M } & \multicolumn{9}{|c|}{ Cephalometric indicators } \\
\hline & SN_PRN & $A L \_A L$ & $\mathrm{CHI}$ _CHI & EK_EK & MF_MF & N_l & PGO_GN & IGO_GN & IN_GARS \\
\hline 16HRZ1 & -0.28 & 0.12 & 0.04 & -0.16 & -0.09 & 0.14 & -0.05 & -0.15 & 0.09 \\
\hline 16HRZ2 & -0.28 & 0.15 & -0.01 & 0.19 & -0.08 & -0.23 & 0.15 & 0.14 & -0.37 \\
\hline $16 \mathrm{HRZ3}$ & -0.18 & 0.15 & -0.06 & -0.16 & -0.09 & -0.17 & 0.03 & -0.03 & 0.00 \\
\hline 16VO_S & $\underline{-0.30}$ & 0.10 & -0.05 & -0.22 & -0.01 & -0.39 & -0.12 & -0.13 & -0.12 \\
\hline 16VO_K & -0.06 & 0.16 & 0.11 & -0.15 & 0.08 & -0.20 & -0.12 & -0.09 & -0.08 \\
\hline 16MD_S & -0.12 & 0.17 & -0.04 & -0.37 & -0.06 & -0.25 & -0.04 & -0.04 & 0.06 \\
\hline 16MD_K & -0.20 & 0.21 & 0.15 & -0.17 & 0.04 & -0.09 & 0.10 & 0.13 & -0.08 \\
\hline $26 \mathrm{HZ}$ & -0.16 & 0.10 & 0.04 & -0.14 & -0.16 & -0.27 & -0.04 & -0.15 & -0.03 \\
\hline $26 \mathrm{HKZ}$ & 0.02 & -0.09 & 0.23 & 0.14 & 0.18 & 0.06 & 0.11 & -0.03 & 0.11 \\
\hline 26HRZ1 & 0.09 & 0.04 & 0.14 & -0.21 & 0.08 & -0.03 & -0.24 & -0.32 & 0.18 \\
\hline 26HRZ2 & -0.15 & -0.13 & -0.18 & 0.10 & -0.21 & -0.08 & 0.09 & 0.00 & -0.13 \\
\hline $26 \mathrm{HRZ3}$ & -0.02 & 0.15 & -0.08 & -0.19 & -0.03 & -0.17 & -0.03 & -0.08 & 0.11 \\
\hline 26VO_K & -0.25 & 0.07 & -0.02 & -0.24 & -0.02 & -0.38 & -0.23 & -0.22 & -0.10 \\
\hline 26VO_S & -0.04 & 0.20 & 0.14 & -0.09 & 0.13 & -0.15 & -0.11 & -0.07 & -0.08 \\
\hline 26MD_K & -0.01 & 0.06 & -0.13 & -0.42 & -0.11 & -0.29 & -0.14 & -0.15 & 0.14 \\
\hline 26MD_S & -0.09 & 0.05 & -0.06 & -0.26 & -0.03 & -0.16 & -0.09 & 0.00 & 0.13 \\
\hline $27 \mathrm{HZ}$ & 0.06 & 0.13 & -0.11 & -0.21 & -0.03 & -0.10 & -0.03 & -0.15 & 0.13 \\
\hline $27 \mathrm{HKZ}$ & 0.14 & -0.07 & 0.00 & -0.22 & 0.10 & 0.11 & -0.13 & -0.29 & $\underline{0.34}$ \\
\hline 27HRZ1 & $\underline{0.30}$ & 0.14 & 0.04 & -0.17 & 0.15 & 0.16 & -0.06 & -0.20 & $\underline{0.33}$ \\
\hline 27HRZ2 & 0.22 & 0.06 & 0.05 & 0.12 & 0.00 & 0.07 & 0.17 & 0.02 & -0.01 \\
\hline 27HRZ3 & -0.19 & 0.24 & -0.28 & -0.18 & -0.09 & -0.16 & -0.13 & -0.25 & -0.09 \\
\hline 27VO_K & -0.06 & 0.17 & 0.02 & -0.37 & -0.08 & -0.22 & -0.26 & -0.30 & -0.01 \\
\hline 27VO_S & -0.01 & 0.01 & 0.18 & -0.27 & 0.22 & 0.03 & -0.09 & -0.16 & -0.03 \\
\hline 27MD_K & -0.03 & 0.16 & 0.11 & -0.17 & 0.04 & -0.20 & 0.01 & -0.04 & -0.13 \\
\hline 27MD_S & 0.00 & 0.11 & 0.12 & -0.18 & 0.11 & -0.10 & 0.07 & 0.02 & -0.08 \\
\hline
\end{tabular}

Notes: here and in the following tables, SN PRN - the depth of the nose; AL AL - width of the base of the nose (distance between alar points); $\mathrm{CHI}$ _CHI - width of the mouth; EK_EK - extraocular width (biorbital width); MF_MF - interosseous width (anterior interorbital width); N_I - distance between the nasion and the interincisor point; RGO_GN - body length of the lower jaw on the right; LGO_GN - body length of the lower jaw on the left; IN_GARS - Garson's morphological index.

Table 6. Correlations of the sizes of $\mathrm{M}$ of a mandible with cephalometric indicators of a facial skull of men of the southern region of Ukraine $(n=6-33)$.

\begin{tabular}{|c|c|c|c|c|c|c|c|c|c|}
\hline \multirow{2}{*}{ Sizes of M } & \multicolumn{10}{|c|}{ Cephalometric indicators } \\
\cline { 2 - 11 } & SN_PRN & AL_AL & CHI_CHI & EK_EK & MF_MF & N_I & PGO_GN & IGO_GN & IN_GARS \\
\hline 47HZ & 0.04 & 0.00 & 0.05 & -0.26 & -0.12 & -0.07 & -0.02 & -0.12 & 0.23 \\
\hline 47HKZ & 0.18 & -0.11 & 0.12 & -0.18 & -0.02 & 0.10 & 0.01 & -0.07 & $\underline{0.31}$ \\
\hline 47HRZ4 & 0.08 & -0.02 & 0.23 & -0.15 & 0.07 & -0.05 & 0.03 & -0.04 & 0.21 \\
\hline 47HRZ5 & -0.19 & 0.21 & -0.21 & -0.28 & -0.27 & -0.10 & -0.08 & -0.20 & 0.11 \\
\hline 47VO_K & -0.18 & 0.17 & -0.01 & -0.16 & -0.19 & -0.29 & -0.06 & -0.10 & -0.15 \\
\hline 47VO_S & -0.01 & -0.01 & -0.02 & -0.27 & 0.01 & -0.07 & -0.16 & -0.22 & 0.08 \\
\hline 47MD_K & 0.15 & -0.05 & 0.12 & -0.24 & -0.03 & -0.02 & -0.10 & -0.11 & 0.15 \\
\hline 47MD_S & 0.17 & 0.04 & 0.14 & -0.13 & 0.17 & 0.06 & 0.01 & 0.01 & 0.19 \\
\hline 46HZ & -0.12 & $\underline{-0.30}$ & 0.00 & -0.11 & -0.06 & -0.19 & -0.05 & -0.20 & 0.06 \\
\hline
\end{tabular}


Soboń J. S., Cherkasova O. V., Gunas V. I., Babych L. V., Kotsyura O. O.

\section{Continuation of table 6 .}

\begin{tabular}{|c|c|c|c|c|c|c|c|c|c|}
\hline \multirow{2}{*}{ Sizes of M } & \multicolumn{9}{|c|}{ Cephalometric indicators } \\
\hline & SN_PRN & AL_AL & $\mathrm{CHI} \mathrm{CHI}$ & EK_EK & MF_MF & N_l & PGO_GN & IGO_GN & IN_GARS \\
\hline $46 \mathrm{HKZ}$ & 0.21 & -0.04 & 0.14 & 0.11 & 0.20 & 0.07 & -0.07 & -0.23 & 0.08 \\
\hline $46 \mathrm{HRZ4}$ & 0.21 & -0.24 & 0.11 & -0.19 & 0.05 & 0.00 & -0.19 & -0.32 & 0.16 \\
\hline 46HRZ5 & -0.41 & -0.08 & -0.24 & -0.26 & -0.23 & -0.26 & -0.03 & 0.01 & 0.00 \\
\hline 46VO_K & 0.06 & 0.14 & 0.00 & -0.17 & -0.03 & -0.14 & -0.13 & -0.12 & 0.01 \\
\hline 46VO_S & 0.13 & 0.30 & 0.09 & 0.00 & 0.22 & 0.08 & -0.11 & -0.14 & 0.01 \\
\hline 46MD_K & 0.21 & 0.00 & 0.06 & -0.20 & 0.00 & 0.10 & -0.07 & -0.06 & 0.10 \\
\hline 46MD_S & 0.19 & 0.16 & 0.05 & -0.15 & 0.12 & -0.01 & -0.16 & -0.22 & 0.12 \\
\hline $36 \mathrm{HZ}$ & 0.00 & $\underline{-0.32}$ & -0.08 & -0.07 & 0.01 & -0.26 & -0.10 & -0.19 & 0.12 \\
\hline $36 \mathrm{HKZ}$ & 0.17 & -0.18 & 0.11 & 0.00 & 0.23 & -0.01 & -0.06 & -0.17 & 0.15 \\
\hline $36 \mathrm{HRZ4}$ & 0.14 & -0.34 & -0.01 & -0.10 & 0.05 & -0.16 & -0.22 & -0.30 & 0.12 \\
\hline $36 \mathrm{HRZ5}$ & -0.38 & -0.04 & -0.42 & -0.21 & -0.35 & -0.25 & -0.16 & -0.16 & 0.03 \\
\hline 36VO_K & -0.11 & 0.21 & -0.02 & -0.17 & -0.03 & -0.27 & -0.17 & -0.23 & -0.17 \\
\hline 36VO_S & 0.12 & 0.20 & -0.10 & -0.15 & -0.03 & -0.09 & -0.16 & -0.18 & 0.05 \\
\hline 36MD_K & 0.15 & -0.01 & 0.03 & -0.10 & -0.05 & -0.14 & -0.09 & -0.08 & -0.02 \\
\hline 36MD_S & 0.09 & 0.10 & 0.10 & -0.16 & 0.11 & -0.17 & -0.06 & -0.09 & 0.03 \\
\hline $37 \mathrm{HZ}$ & 0.13 & 0.02 & -0.02 & -0.23 & -0.12 & -0.10 & -0.14 & -0.23 & 0.26 \\
\hline $37 \mathrm{HKZ}$ & 0.19 & -0.02 & 0.14 & -0.11 & 0.02 & 0.00 & -0.04 & -0.07 & 0.20 \\
\hline 37HRZ4 & 0.10 & -0.17 & 0.10 & -0.11 & 0.11 & -0.21 & -0.08 & -0.13 & 0.18 \\
\hline 37HRZ5 & -0.13 & 0.18 & -0.26 & -0.33 & -0.26 & -0.08 & -0.13 & -0.30 & 0.20 \\
\hline 37VO_K & -0.16 & 0.08 & -0.09 & -0.23 & -0.26 & -0.21 & -0.10 & -0.20 & -0.07 \\
\hline 37VO_S & -0.06 & -0.04 & -0.03 & -0.25 & -0.10 & -0.02 & -0.06 & -0.14 & 0.04 \\
\hline 37MD_K & 0.15 & -0.19 & 0.10 & -0.30 & -0.02 & 0.01 & 0.05 & 0.02 & 0.11 \\
\hline 37MD_S & 0.23 & -0.01 & 0.19 & -0.12 & 0.20 & 0.12 & 0.07 & 0.12 & 0.14 \\
\hline
\end{tabular}

the southern administrative-territorial region of Ukraine are presented in Tables 1-6.

\section{Discussion}

Orthodontics and anthropology have become inseparable scientific fields since it became clear that without maintaining the correct proportions of the face and teeth, it is impossible to achieve a perfect, "Hollywood" smile [7, 21]. In this context, the dental industry cannot continue to develop separately, despite the cephalometric guidelines and, most importantly, the identification of their relationships with odontometric within different ethnic or regional groups.

Clearly, there is no doubt that different ethnic groups and nationalities have different characteristics of cephalometric and odontometric indicators and different characteristics of the relationships between them. Thus, when comparing these indicators in women of Saudi Arabia and Japan, it was found that the former have significantly higher rates of gonial angle, facial angle, lower facial height, more protruding incisors and nose, protruding chin than the latter [1]. Also significant differences in the above indicators were found when comparing the data of Syrian and Hungarian adolescents [2], residents of India and Malaysia [10]. Vela E. and co-authors [25] found that there are differences between US residents of European and Mexican descent.

Boujoual I. and others [4, 5] surveyed 200 people in Morocco, and after statistical processing of the data found a strong correlation between the shape of the face and the shape of the central incisor of the upper jaw.

It has been established that the thickness of the gums is closely related to the index of the human face and the angulation of the anterior part of the lower jaw [11].

Linjawi A. I. [12, 13] in the examination of 210 patients aged 11-15 years and subsequent statistical data processing found strong correlations between the state of molar health and such indicators as sex, vertical growth pattern and overbite, but no correlations with cephalometric indicators. Similar data were also obtained by a group of Indian researchers [16].

At the same time, significant correlations between cephalometric parameters and odontometric parameters have been found by Greek researchers [18]. Thus, in their study, they found the following statistical relationship 
between the indicators of covariance of the lower first molar and craniofacial complex in prepubertal and adult groups of subjects ( $R V=14.05 \%, p=0.0099$ and $R V=12.31 \%$, $\mathrm{p}=0.0162$, respectively).

As a result of the analysis of reliable and average strength of unreliable correlations of computer-tomographic sizes of $M$ with cephalometric indicators and indices of practically healthy men from the southern administrativeterritorial region of Ukraine multiple character of mostly direct reliable $(r=0.35-0.56)$ and average strength of unreliable $(r=0.30-0.34)$ correlations were found only between most sizes of $M$ on the lower jaw and the length and height of the nose.

The quantitative analysis of reliable and average strength of unreliable correlations of computer-tomographic sizes of $\mathrm{M}$ with cephalometric indicators and indices of practically healthy men of the southern administrativeterritorial region of Ukraine revealed the following distribution of correlations:

between the upper $M$ and indicators of a brain skull 21 correlations from 252 possible (8.3\%), from which, $0.4 \%$ of direct reliable average force, $3.2 \%$ of return reliable average force and $4.8 \%$ of return unreliable average force; among which - with the first M 9 correlations from 126 possible $(0.8 \%$ of direct reliable average force, $3.2 \%$ of reverse reliable average force and $3.2 \%$ of reverse unreliable average force); with the second $\mathrm{M} 12$ correlations out of 126 possible (3.2\% of reversible reliable medium strength and $6.3 \%$ of reverse unreliable medium strength); with the height of the teeth, their crowns and the length of the roots 16 correlations out of 140 possible $(0.7 \%$ of direct reliable medium force, $4.3 \%$ of inverse reliable medium force and $6.4 \%$ of inverse unreliable medium force); with vestibular-lingual and mesio-distal dimensions 5 correlations out of 112 possible $(1.8 \%$ of reverse reliable mean forces and $2.7 \%$ of inverse unreliable middle forces);

between lower $M$ and cranial skull indicators only 6 correlations out of 224 possible (2.7\%), of which, $0.9 \%$ of reversible reliable mean force and $1.8 \%$ of inverse unreliable average force; among which - with the first M 2 correlations from 112 possible $(0.9 \%$ of reverse reliable average force and $0.9 \%$ of reverse unreliable average force); with the second M 4 correlations from 112 possible $(0.9 \%$ of return reliable average force and $2.7 \%$ of return unreliable average force); with the height of the teeth, their crowns and the length of the roots 6 correlations out of 112 possible $(1.8 \%$ of reverse reliable medium strength and $3.6 \%$ of inverse unreliable medium strength); with vestibular-lingual and mesio-distal dimensions - reliable or medium-strength unreliable correlations are not established at all;

between the upper $M$ and facial skull indicators 48 correlations out of 648 possible $(7.4 \%)$, of which, $0.2 \%$ direct reliable medium strength, $1.7 \%$ direct unreliable medium strength, $2.8 \%$ reverse reliable medium strength and $2.8 \%$ reverse unreliable medium strength; among which - with the first M 26 correlations from 324 possible ( $4.9 \%$ of reverse reliable average force and $3.1 \%$ of reverse unreliable average force); with the second M 22 correlations from 324 possible $(0.3 \%$ of direct reliable average force, $3.4 \%$ of direct unreliable average force, $0.6 \%$ of reverse reliable average force and $2.5 \%$ of reverse unreliable average force); with the height of the teeth, their crowns and the length of the roots 23 correlations out of 360 possible ( $1.7 \%$ of direct unreliable medium strength, $2.5 \%$ of inverse reliable of medium strength and $2.2 \%$ of inverse unreliable medium strength); with vestibular-lingual and mesio-distal dimensions 25 correlations out of 288 possible $(0.3 \%$ of direct reliable medium forces, $1.7 \%$ of direct unreliable medium forces, $3.1 \%$ of inverse reliable medium forces and $3.5 \%$ of inverse unreliable medium forces);

between lower $M$ and facial skull indicators 76 correlations out of 576 possible (13.2\%), of which, $3.6 \%$ direct reliable medium strength, $3.6 \%$ direct unreliable medium strength, $3.6 \%$ inverse reliable medium strength and $2.3 \%$ inverse unreliable medium strength; among which - with the first M 42 correlations from 288 possible (3.1\% of direct reliable average force, $4.2 \%$ of direct unreliable average force, $5.2 \%$ of reverse reliable average force and $2.1 \%$ of reverse unreliable average force); with the second M 34 correlations from 288 possible (4.2\% of direct reliable average force, $3.1 \%$ of direct unreliable average force, $2.1 \%$ of reverse reliable average force and $2.4 \%$ of reverse unreliable average force); with the height of the teeth, their crowns and the length of the roots 42 correlations out of 288 possible $(1.4 \%$ of direct reliable medium strength, $3.5 \%$ of direct unreliable medium strength, $5.9 \%$ of inverse reliable medium strength and $3.8 \%$ of inverse unreliable medium strength); with vestibular-lingual and mesio-distal dimensions 34 correlations out of 288 possible $(5.9 \%$ of direct reliable medium forces, $3.8 \%$ of direct unreliable medium forces, $1.4 \%$ of reverse reliable medium forces and $0.7 \%$ of inverse unreliable medium forces).

The obtained results of qualitative and quantitative analysis of correlations of computer-tomographic sizes of $M$ with cephalometric indicators and indices of practically healthy men from the southern administrative-territorial region of Ukraine have many numerical differences from the results received at the analysis of correlations of computer-tomographic sizes of $\mathrm{M}$ with cephalometric indicators and indices of practically healthy men from the northern region of Ukraine [17].

Thus, our analysis of the correlations of computed tomography dimensions of the $\mathrm{M}$ with cephalometric indicators and indices confirms the population specificity of the features of the dental-jaw system.

\section{Conclusions}

1. In practically healthy men of the southern region of Ukraine the peculiarities of correlations of linear computed tomographic sizes of $\mathrm{M}$ with cephalometric indicators of 
cerebral and facial skull are established. In the qualitative analysis, the multiple nature of the correlations was found only between the majority of the size of the $\mathrm{M}$ on the lower jaw and the length and height of the nose (mostly direct reliable and medium strength unreliable).

2. In quantitative analysis, the majority of reliable correlations were found between the computed tomography size of the $\mathrm{M}$ and the size of the face $(7.4 \%$ on the upper jaw, most of which are inverse, and on the lower jaw - $13.2 \%$, almost evenly direct and inverse); according with the indicators of the skull - on the upper jaw $8.3 \%$, almost all of which have the opposite character, and on the lower jaw

\section{References}

[1] Abbassy, M. A., \& Abushal, A. (2015). Differences in dentofacial characteristics of Class I malocclusion between Saudi and Japanese adult females. Journal of orthodontic science, 4(3), 86-91. doi: $10.4103 / 2278-0203.160244$

[2] Al Ayoubi, A., Dezfully, A. K., \& Madlena, M. (2020). Dentoskeletal and tooth-size differences between Syrian and Hungarian adolescents with Class II division 1 malocclusion: a retrospective study. BMC Research Notes, 13(1), 1-7. doi: 10.1186/s13104-020-05115-0

[3] Baron, C., Houchmand-Cuny, M., Enkel, B., \& Lopez-Cazaux, S. (2018). Prevalence of dental anomalies in French orthodontic patients: A retrospective study. Archives de Pediatrie, 25(7), 426-430. doi: 10.1016/j.arcped.2018.07.002

[4] Boujoual, I., Mouhibi, A., Jouhadi, E. M., \& Andoh, A. (2018). Correlation between the form of the maxillary central incisor and the inverted form of the face (Part I: literature background). International Journal of Information Research and Review, 5(10), 5766-5769.

[5] Boujoual, I., Mouhibi, A., Mbarki, E., \& Andoh, A. (2018). Correlation between the form of the maxillary central incisor and the inverted form of the face (Part II: statistical study on a Moroccan sample). International Journal of Information Research and Review, 5(10), 5770-5773.

[6] Bunak, V. V. (1941). Антропометрия. Практический курс [Anthropometry: a practicalcourse]. М.: Учпедгиз - М.: Uchpedgiz.

[7] Celebi, A. A., Kau, C. H., \& Ozaydin, B. (2017). Three-dimensional anthropometric evaluation of facial morphology. Journal of Craniofacial Surgery, 28(5), e470-e474. doi: 10.1097/ SCS. 0000000000003773

[8] Dang, H. Q., Constantine, S., \& Anderson, P. J. (2017). The prevalence of dental anomalies in an Australian population. Australian dental journal, 62(2), 161-164. doi: 10.1111/ adj.12443

[9] Gayathri, M., \& Arun, A. V. (2018). Relationship between nasolabial angle and maxillary incisor proclination in South Indian population. Drug Invention Today, 10, 1-4.

[10] Jain, A. R. (2018). Comparison of facial form and tooth form between South Indian and Malaysian edentulous patients. Drug Invention Today, 10(6), 874-879.

[11] Kolte, R. A., Kolte, A. P., Kharkar, V. V., \& Bawankar, P. (2020). Influence of facial index, facial profile, lip size, and angulations of teeth on gingival characteristics of anterior teeth: A genderbased evaluation. Journal of Esthetic and Restorative Dentistry. doi: $10.1111 /$ jerd. 12600

[12] Linjawi, A. I. (2016). Age-and gender-related incisor changes in different vertical craniofacial relationships. Journal of orthodontic science, 5(4), 132-137. doi: 10.4103/2278- only $2.7 \%$, all have the opposite character.

3. It was found that with the indicators of the brain skull, the relative majority of reliable correlations are observed with the height of the teeth, their crowns and the length of the roots (on the upper jaw $11.4 \%$, mostly reverse; on the lower jaw $5.4 \%$, all reverse); and with the facial skull almost evenly with the height of the teeth, their crowns and the length of the roots (on the upper jaw $6.4 \%$, mostly reverse; on the lower jaw $14.6 \%$, mostly reverse) and with vestibular-lingual and mesio-distal dimensions (on the upper jaw $8.7 \%$, mostly reverse, on the lower jaw $11.8 \%$, mostly straight).

\subsection{6}

[13] Linjawi, A. I. (2016). First molar health status in different craniofacial relationships. Clinical, cosmetic and investigational dentistry, 8, 89-94. doi: 10.2147/CCIDE.S108670

[14] Mahn, E., Walls, S., Jorquera, G., Valdis, A. M., Val, A., \& Sampaio, C. S. (2018). Prevalence of tooth forms and their gender correlation. Journal of Esthetic and Restorative Dentistry, 30(1), 45-50. doi: 10.1111/jerd.12341

[15] Patil, S., Doni, B., Kaswan, S., \& Rahman, F. (2013). Prevalence of dental anomalies in Indian population. Journal of clinical and experimental dentistry, 5(4), e183-e186. doi: 10.4317/ jced.51119

[16] Pillai, J. P., Patel, R. A., Banker, A. M., \& Rajarajeswari, J. (2018). Correlation between maxillary central incisor crown form and maxillary dental arch form: A Model-Based morphometric, cross-sectional study. Journal of Forensic Science and Medicine, 4(2), 70-75. doi: 10.4103/ jfsm.jfsm_59_17

[17] Polishchuk S. S., Kotsyura O. O., Dmitriev M. O., Orlovskiy V. O., \& Popov M. V. (2020). Correlations of linear sizes of molars with cephalometric indicators of practically healthy men of the northern region of Ukraine. Reports of Morphology, 26(1), 37-47. doi: 10.31393/morphology-journal-2020-26(1)-06

[18] Polychronis, G., \& Halazonetis, D. J. (2014). Shape covariation between the craniofacial complex and first molars in humans. Journal of anatomy, 225(2), 220-231. doi: 10.1111/joa.12202

[19] Proffit, U. R. (translation from English; Ed. L. S. Persina) (2015). Современная ортодонтия [Modern orthodontics]. М.: МЕДпресс-инфрорм - M.: Medpress-inform.

[20] Ramiro-Verdugo, J., De Vicente-Corominas, E., MontielCompany, J. M., Gandia-Franco, J. L., \& Bellot-Arcis, C. (2015). Association between third molar agenesis and craniofacial structure development. American Journal of Orthodontics and Dentofacial Orthopedics, 148(5), 799-804. doi: 10.1016/ j.ajodo.2015.05.025

[21] Rossetti, A., De Menezes, M., Rosati, R., Ferrario, V. F., \& Sforza, C. (2013). The role of the golden proportion in the evaluation of facial esthetics. The Angle Orthodontist, 83(5), 801-808. doi: $10.2319 / 111812-883.1$

[22] Sakulratchata, R., Wongma, S., Saenmood, S., Thunyanut rianpingwang, \& Chiohanangkun, S. (2020). Prevalence and Characteristics of Dental Anomalies in Pediatric Patients at a Dental Hospital in Thailand. Naresuan University Journal: Science and Technology (NUJST), 29(2), 73-83.

[23] Samusev, R. P., Krayushkin, A. I., \& Dmitrienko, S. V. (Ed. M. R. Sapin) (2002). Основы клинической морфологии зубов: учебное пособие [Basics of clinical morphology of teeth: a tutorial]. М. : ООО "Издательский дом "ОНИКС 21 век": 
ООО "Мир и Образование" - М: ООО "Publishing House ONIX21 vek": OOO "World and Education".

[24] Seden, A., \& Defne K. Lower Incisor Inclination in Relation to Head Posture, Tongue and Hyoid Positions. Cumhuriyet Dental Journal, 22(3), 310-317. doi: 10.7126/cumudj.553220

[25] Vela, E., Taylor, R. W., Campbell, P. M., \& Buschang, P. H. (2011). Differences in craniofacial and dental characteristics of adolescent Mexican Americans and European Americans.
American journal of orthodontics and dentofacial orthopedics, 140(6), 839-847. doi: 10.1016/j.ajodo.2011.04.026

[26] Zubov, А. А. (2003). Одонтология. Методика антропологических исследований [Odontology. Methodology of anthropological research]. М.: Наука - М.: Science. 